\title{
Spatial Dependence of Magnetization and Gaussian Fluctuations of Molecular Field in an Ising Nanopyramid Interacting with the Substrate
}

\author{
L.S. BORKOWSKI AND Z. JACYNA-ONYSZKIEWICZ \\ Faculty of Physics, A. Mickiewicz University \\ Umultowska 85, 61-614 Poznań, Poland
}

\begin{abstract}
We study thermodynamic properties of an Ising model of a ferromagnetic nanoscopic pyramid deposited on a ferromagnetic bulk substrate. The influence of the interaction between the pyramid and the substrate is calculated in terms of the reduced-state (density) operator used for description of thermodynamic properties of nanoscopic systems. The spatial distribution of magnetization in the nanopyramid is obtained in the Gaussian fluctuations approximation.
\end{abstract}

PACS numbers: $75.75 .+\mathrm{a}$

\section{Introduction}

Magnetic nanoparticles studied experimentally are often deposited on a bulk substrate. In theoretical descriptions of these experiments the coupling with the substrate is often neglected. We show how such interaction can be accounted for in the ferromagnetic Ising model using the reduced-state (density) operator [1]. The calculations are carried out within the Gaussian fluctuations approximation (GFA). GFA is a modified version of the high density expansion as proposed in Ref. [2].

The new element of GFA is the self-consistent summation of partial sums of a certain class of the Feynman diagrams at each stage of the calculations. This procedure removes earlier difficulties such as e.g. a complex Curie temperature [3].

\section{Theory and results}

We consider magnetic properties of a nanopyramid of spins- $1 / 2$ on described by the spin Hamiltonian

$$
H=-\frac{1}{2} I \sum S_{\boldsymbol{f} l}^{z} S_{\boldsymbol{f}^{\prime} l^{\prime}}^{z}
$$

where $I$ is the coupling parameter and $\sum$ stands for summation over pairs of nearest neighbors on a simple cubic lattice, $\boldsymbol{f}$ denotes two-dimensional position vectors of a spin belonging to a given monoatomic layer $l=1,2,3,4$ of the pyramid. There are 4 spins in the $l=1$ layer, 16 spins in the $l=2$ layer, 36 spins in the $l=3$ layer and 64 spins in the $l=4$ layer. The total number of spins in the pyramid is 120 (see Fig. 1).

The nanopyramid interacts with bulk ferromagnetic substrate. The interaction within the substrate has the form

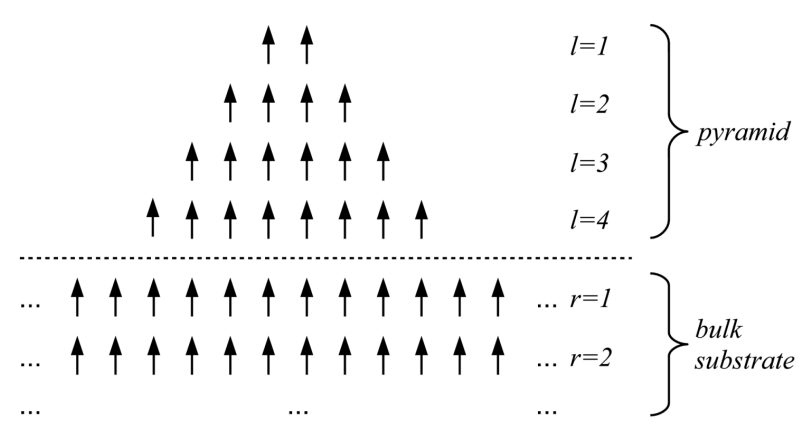

Fig. 1. Schematic diagram of ferromagnetic nanoscopic pyramid deposited on a ferromagnetic substrate.

$$
H_{\mathrm{s}}=-\frac{1}{2} I_{1} \sum S_{\boldsymbol{g}_{r}}^{z} S_{\boldsymbol{g}^{\prime} r^{\prime}}^{z}
$$

Here $\boldsymbol{g}$ denotes the two-dimensional position vectors of spin belonging to a monoatomic layer $r=1,2, \ldots$ The interaction of the nanopyramid with the substrate is described by the Heisenberg Hamiltonian

$$
H_{I}=-\frac{1}{2} I_{2} \sum_{\boldsymbol{f g}} \boldsymbol{S}_{\boldsymbol{f l = 4}} \boldsymbol{S}_{\boldsymbol{g} r=1} .
$$

Using the equilibrium reduced-state operator [1], with the assumption

$$
\left|\left\langle S_{\boldsymbol{g r}}^{x}\right\rangle\right|,\left|\left\langle S_{\boldsymbol{g}_{r}}^{y}\right\rangle\right| \ll\left|\left\langle S_{\boldsymbol{g r} r}^{z}\right\rangle\right|, \quad I_{2} \ll I_{1},
$$

we obtain the nanopyramid-substrate interaction term

$$
H^{\prime}(\beta)=-\frac{1}{2} I_{2}\left\langle S_{r=1}^{z}\right\rangle \sum_{\boldsymbol{f}} S_{\boldsymbol{f} l=4}^{z},
$$

where 


$$
\left\langle S_{r=1}^{z}\right\rangle=\operatorname{Tr}_{\mathrm{s}}\left(S_{\boldsymbol{g} r=1}^{z} \exp \left(\beta\left(F_{\mathrm{s}}-H_{\mathrm{s}}\right)\right)\right)
$$

is the average bulk substrate spin moment in the layer $r=1$. After introducing the following reduced magnitudes:

$$
\begin{aligned}
X_{\boldsymbol{f} l} & =2\left\langle S_{\boldsymbol{f} l}^{z}\right\rangle, \quad y_{r}=2\left\langle S_{r}^{z}\right\rangle_{0}, \quad t=\frac{4}{\beta I}, \\
a & =\frac{I_{2}}{I}, \quad b=\frac{I_{1}}{I},
\end{aligned}
$$

and some manipulations, we can write the final equations in a compact form,

$$
X_{\boldsymbol{f} l}=\frac{1}{\sqrt{2 \pi}} \int_{-\infty}^{+\infty} \mathrm{e}^{-u^{2} / 2} \tanh \left(W_{\boldsymbol{f} l}+u \delta W_{\boldsymbol{f} l}\right) \mathrm{d} u,
$$

where

$$
\begin{aligned}
W_{\boldsymbol{f} l}= & \frac{1}{t}\left(\sum_{\boldsymbol{f}^{\prime} l^{\prime}} X_{\boldsymbol{f}^{\prime} l^{\prime}}+a \delta_{l, 4} y_{r=1}\right) \\
\delta W_{\boldsymbol{f} l} & =\frac{2}{t}\left\{\frac { 1 } { \sqrt { 2 \pi } } \int _ { - \infty } ^ { + \infty } \mathrm { e } ^ { - u ^ { 2 } / 2 } \left[1-\tanh ^{2}\left(W_{\boldsymbol{f} l}+u \delta W_{\boldsymbol{f} l}\right)\right.\right. \\
& +\sum_{\boldsymbol{f}^{\prime} l^{\prime}}\left(1-\tanh ^{2}\left(W_{\boldsymbol{f}^{\prime} l^{\prime}}+u \delta W_{\boldsymbol{f}^{\prime} l^{\prime}}\right)\right) \\
& \left.\left.+a^{2} \delta_{l, 4}\left(1-\tanh ^{2}\left(V_{r}+u \delta V_{r}\right)\right)\right] \mathrm{d} u\right\}^{1 / 2} \\
V_{r}= & \frac{b}{t}\left[4 y_{r}+y_{r+1}+\left(1-\delta_{r, 1}\right) y_{r-1}\right] \\
y_{r}= & \frac{1}{\sqrt{2 \pi}} \int_{-\infty}^{+\infty} \mathrm{e}^{-u^{2} / 2} \tanh \left(V_{r}+u \delta V_{r}\right) \mathrm{d} u \\
\delta V_{r}= & \frac{2 b}{t}\left\{\frac { 1 } { \sqrt { 2 \pi } } \int _ { - \infty } ^ { + \infty } \mathrm { e } ^ { - u ^ { 2 } / 2 } \left[4\left(1-\tanh ^{2}\left(V_{r}+u \delta V_{r}\right)\right)\right.\right. \\
& +\left(1-\delta_{r, 1}\right)\left(1-\tanh ^{2}\left(V_{r-1}+u \delta V_{r-1}\right)\right) \\
+ & \left.\left.\left(1-\tanh ^{2}\left(V_{r+1}+u \delta V_{r+1}\right)\right)\right] \mathrm{d} u\right\}^{1 / 2}
\end{aligned}
$$

where $\delta W_{\boldsymbol{f l}}$ and $\delta V_{r}$ are the mean Gaussian fluctuations of the molecular fields $W_{\boldsymbol{f} l}$ and $V_{r}$, respectively. Equations (8)-(13) were solved numerically.

Figure 2 presents spatial distributions of Gaussian fluctuations of molecular field $\delta W_{\boldsymbol{f} l}$ for $l=4$ at $t=4.0$ and $a=0.5, b=1.0$. In the pyramid layer adjacent to the substrate the magnitude of fluctuations is largest in the second row of atoms, counting from the outside. Maximum fluctuations occur along the edges of the pyramid. Smaller fluctuations of magnetic moments in the center of the base of the apex is an effect of the substrate. Figure 3 shows magnetization of individual layers of the pyramid and the substrate. The influence of the pyramid extends several layers into the substrate. Similar conclusion was reached in an earlier study [4] of the interaction between a magnetic nanotip and a magnetic surface using the tightbinding model. The tight-binding calculation for a Fe tip on a Fe surface shows that only the first four layers of the tip support are affected. The inclusion of fluctuations in

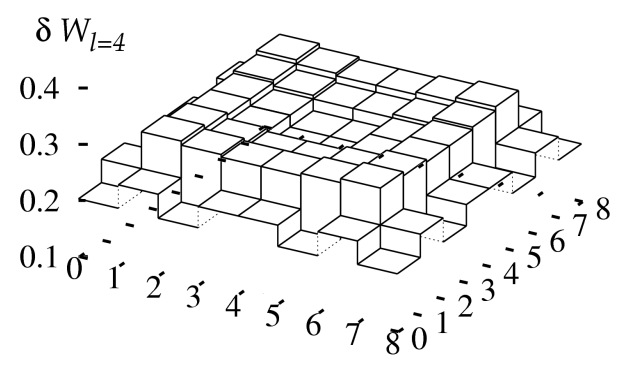

Fig. 2. Spatial distribution of Gaussian fluctuations of the molecular field in pyramid layer $l=4$ at temperature $t=4.0$ and $a=0.5, b=1$.

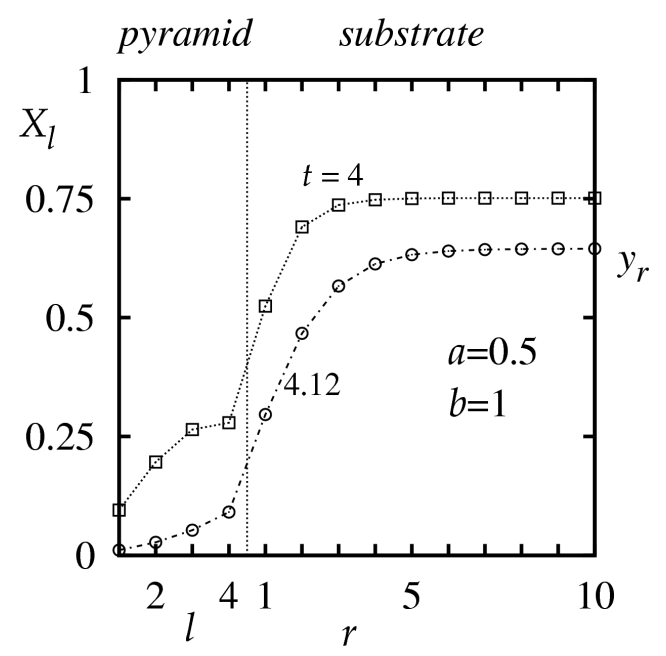

Fig. 3. Magnetization in individual layers of the pyramid $X_{l}$ and the substrate $y_{r}$ for $a=0.5, b=1.0$ and $t=4.0,4.12$.

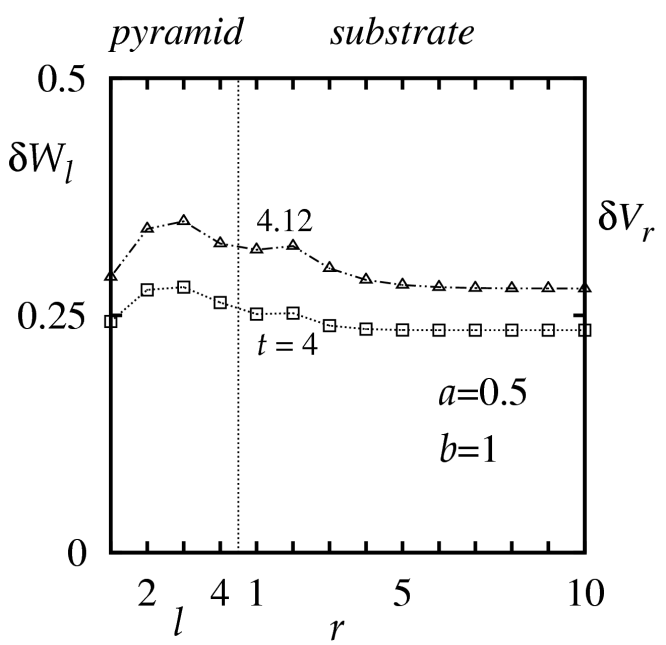

Fig. 4. Gaussian fluctuation of the molecular field in individual layers of the pyramid and the substrate for $a=0.5, b=1.0$ and $t=4.0,4.12$. 
our work increase the depth of this influence as $t \rightarrow t_{\mathrm{c}}$.

Figure 4 presents the mean Gaussian fluctuation of molecular field

$$
\delta W_{l}=\frac{\sum_{\boldsymbol{f} l} \delta W_{\boldsymbol{f} l}}{\sum_{\boldsymbol{f} l}}
$$

and $\delta V_{r}$ for the pyramid and the substrate, respectively.

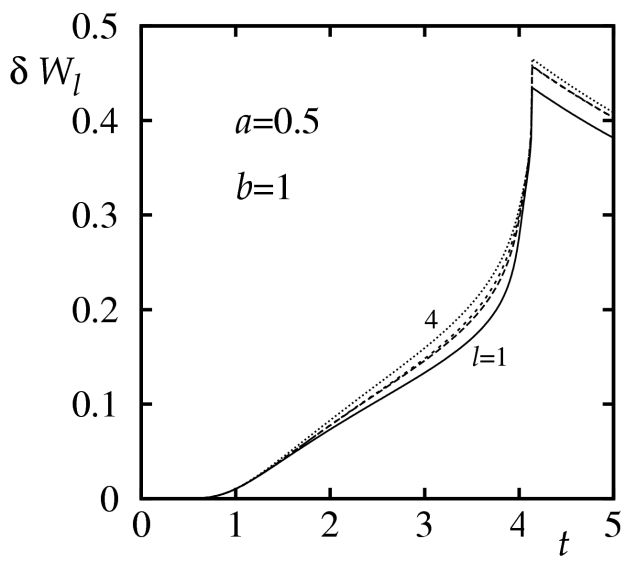

Fig. 5. Temperature dependence of Gaussian fluctuations of molecular field in individual layers of the pyramid $\delta W_{l}$ for $a=0.5$ and $b=1.0$.

Finally, Fig. 5 shows Gaussian fluctuation of the molecular field as a function of temperature.

Our work may be helpful in interpretation of experiments using magnetic exchange force microscopy (MExFM) [5]. Atomically sharp magnetic tips are crucial in achieving lateral high spatial resolution. Therefore, the knowledge of the spatial distribution of fluctuations and its temperature dependence should improve both the calibration of the scanning tunneling microscopy (STM) device and the accuracy of MExFM measurements.

The ability to control magnetic properties of nanoclusters [6] is one of the central problems of nanotechnology. Arrays of nanoclusters are also intensively studied $[7,8]$. The theory presented here may be applied to various geometrical shapes used in experiments [9], e.g. chains of particles, striped and cylindrical nanowires, nanodots, nanojunctions and surface steps.

\section{References}

[1] Z. Jacyna-Onyszkiewicz, Physica A 305, 497 (2002).

[2] Z. Onyszkiewicz, Phys. Lett. A 76, 411 (1980); Z. Onyszkiewicz, Physica A 103, 257 (1980).

[3] Z. Onyszkiewicz, A. Wierzbicki, Phys. Lett. A 116, 335 (1986).

[4] H. Ness, F. Gautier, Phys. Rev. B 52, 7352 (1995).

[5] U. Kaiser, A. Schwarz, R. Wiesendanger, Nature 446, 522 (2007).

[6] C.F. Hirjibehedin, C.P. Lutz, A.J. Heinrich, Science 312, 1021 (2006).

[7] B. Müller, M. Riedel, R. Michel, S.M. De Paul, R. Hofer, D. Heger, D. Grützmacher, J. Vac. Sci. Technol. B 19, 1715 (2001).

[8] M. Jamet, A. Barski, T. Devillers, V. Poydenot, R. Dujardin, P. Bayle-Guillemaud, J. Rothman, E. Bellet-Amalric, A. Marty, J. Cibert, R. Mattana, S. Tatarenko, Nature Mater. 5, 653 (2006).

[9] R. Skomski, J. Phys., Condens. Matter 15, R841 (2003). 